\title{
Cross-Sectional Association between Polyfluoroalkyl Chemicals and Cognitive Limitation in the National Health and Nutrition Examination Survey
}

\author{
Melinda C. Power ${ }^{a, b}$ Thomas F. Webster ${ }^{c}$ Andrea A. Baccarellia, b \\ Marc G. Weisskopfa, b \\ Departments of a Environmental Health and ${ }^{b}$ Epidemiology, Harvard School of Public Health, and \\ 'Department of Environmental Health, Boston University School of Public Health, Boston, Mass., USA
}

\section{Key Words \\ National Health and Nutrition Examination Survey • \\ Cognitive function - Polyfluoroalkyl chemicals • \\ Epidemiology $\cdot$ Risk factor}

\begin{abstract}
Background/Aims: Our limited understanding of how polyfluoroalkyl chemicals (PFCs) may impact on human health suggests the potential for a protective impact on brain health. This study was designed to explore the association between PFCs and cognitive ability in older adults. Methods: We assessed the association between four PFCs, perfluorooctanoic acid (PFOA), perfluorooctane sulfonic acid (PFOS), perfluorononanoic acid (PFNA) and perfluorohexane sulfonic acid (PFHxS), and self-reported limitation due to difficulty remembering or periods of confusion using data from participants aged 60-85 years from the 1999-2000 and 2003-2008 National Health and Nutrition Examination Surveys. We also considered whether diabetic status or diabetic medication use modifies this association in light of in vitro evidence that PFCs may act on the same receptors as some diabetic medications. Results: In multivariable adjusted models, point estimates suggest a protective association between PFCs and self-reported cognitive limitation (odds ra-
\end{abstract}

tio, OR; $95 \%$ confidence interval, $\mathrm{Cl})$ for a doubling in PFC concentration: PFOS (OR, 0.90; 95\% Cl, 0.78, 1.03), PFOA (OR, $0.92 ; 95 \% \mathrm{Cl}, 0.78,1.09), \mathrm{PFNA}(\mathrm{OR}, 0.91 ; 95 \% \mathrm{Cl}, 0.79,1.04)$ and PFHxS (OR, 0.93; $95 \% \mathrm{Cl}, 0.82,1.06)$. The protective association was concentrated in diabetics, with strong, significant protective associations in nonmedicated diabetics. Conclusions: This cross-sectional study suggests that there may be a protective association between exposure to PFCs and cognition in older adults, particularly diabetics.

Copyright $\odot 2012$ S. Karger AG, Basel

\section{Introduction}

Polyfluoroalkyl chemicals (PFCs) are a heterogeneous family of man-made chemicals that structurally resemble fatty acids. PFCs and their precursor compounds are found in products such as stain and oil-resistant coatings, food packaging, and emulsifiers, and have been found in environmental media and in the sera of human populations worldwide [1,2].

Perfluorooctanoic acid (PFOA) and perfluorooctane sulfonic acid (PFOS), two common PFCs, have been shown to activate human peroxisome proliferator-activated receptors (PPARs) in vitro [3]. PPAR- $\gamma$, one of the

\section{KARGER \\ Fax +4161306 1234 \\ E-Mail karger@karger.ch}

www.karger.com (c) 2012 S. Karger AG, Basel

$0251-5350 / 13 / 0402-0125 \$ 38.00 / 0$

Accessible online at:

www.karger.com/ned
Melinda C. Power

Harvard School of Public Health, EOME, Landmark Center, 3rd Floor East PO Box 15697

Boston, MA 02215 (USA)

Tel. +1 617384 8872, E-Mail melindacpower@gmail.com 
three subtypes, has been highlighted as a potential therapeutic target in neurodegenerative diseases, in part because PPAR- $\gamma$ agonists, including thiazolidinedione drugs, which treat diabetes, appear neuroprotective [4]. If PFCs activate human PPAR- $\gamma$ they may similarly protect against cognitive impairment.

PFCs appear to suppress immune function in animal studies, either through or exclusive of PPAR activation [5, 6]. Similarly, in a recent study of the association between vaccine response and PFCs in children, higher PFC exposures were associated with lower immune response [7]. Although in many cases impaired immunity is harmful, inflammation is part of all neurodegenerative diseases, and if PFCs reduce neuroinflammation, we might expect to see a beneficial impact of PFCs on brain health in older adults. Also, in animal models, PFCs have been shown to effect on calcium ion channel function $[8,9]$ and neuroendocrine function [10], although it is difficult to know whether such effects would promote brain health or disease.

This cross-sectional study was designed to assess the potential for an association between PFCs and cognition in older adults. We also explored whether diabetic status or the use of diabetic medications modified the association between PFCs and cognition, as we hypothesized that both PFCs and thiazolidinedione drugs, used to treat diabetes, act through the activation of PPAR- $\gamma$.

\section{Materials and Methods}

\section{Study Population}

The United States National Health and Nutrition Examination Survey (NHANES) uses a complex, multistage probability sampling design to select participants who are nationally representative of the noninstitutionalized United States population. NHANES data and details on survey methods are publically available (http://www.cdc.gov/nchs/nhanes.htm). This study was approved by the National Center for Health Statistics Research Ethics Review Board and all participants provided written informed consent.

We used data from all participants meeting eligibility criteria from the 1999-2000 and the 2003-2004, 2005-2006 and 20072008 continuous NHANES to conduct this study. Each of these cycles had an unweighted participation rate of approximately $80 \%$. Eligibility criteria for inclusion in an analysis required participants to be between the ages of 60 and 85 , to have a valid measure of PFC serum concentrations, and to have data on the outcome measure of interest.

\section{Exposure Assessment}

PFCs were measured in serum from a random one third subset of participants aged 12 and older in 1999-2000 and 2003-2008. We focused on the four most common PFCs that were detected in
$>98 \%$ of the persons sampled: PFOA, PFOS, perfluorononanoic acid (PFNA) and perfluorohexane sulfonic acid (PFHxS). Details of sample collection, storage, quality control and laboratory methods have been described elsewhere [11]. Briefly, all the samples were analyzed using high-performance liquid chromatography/tandem mass spectrometry against calibration standards spiked into calf serum. Quality control measures included analysis of reagent blank, serum blank, and low and high concentration samples derived from calf serum. Coefficients of variation vary by cycle and PFC, but were generally between 10 and 15\% (range, $7-22)[11,12]$. NHANES recoded samples below the limit of detection as the limit of detection divided by $\sqrt{ } 2$.

\section{Outcome Assessment}

We used self-reported limitation (yes/no) due to difficulty remembering or periods of confusion as our primary outcome measure [13]. Proxy respondents were used when the participant was unable to answer for themselves. We considered persons who refused to answer or who replied 'don't know' ( $<3$ people per NHANES cycle) to be missing data. We also considered two additional measures in secondary analyses: self-reported difficulty with activities of daily living due to senility (yes/no) and performance on the Digit-Symbol Substitution Task (DSST) [14]. These were considered secondary outcomes because of the small number of participants reporting difficulty with activities of daily living due to senility $(n=17)$ and the small number of participants with both DSST and PFC measures $(n=275)$. In the DSST, participants used a substitution key to draw symbols under corresponding numbers. The final score is the number of correct symbols drawn in $120 \mathrm{~s}$, with higher scores indicating better performance.

\section{Statistical Analysis}

A small number of persons were missing data on variables selected for inclusion in multivariable models as potential confounders (table 1). Therefore, we used multiple imputation to deal with missing covariate data, leaving imputed values for dichotomous and dummy variables unrounded [15]. We present analyses adjusted for age (continuous and age-squared), race/ethnicity (White, Black, non-Mexican American Hispanic, Mexican American born in Mexico, Mexican American not born in Mexico, and other), gender (male/female), NHANES cycle (19992000, 2003-2004, 2005-2006, 2007-2008), education $(<12,12$, $>12-16,>16$ years), poverty-income ratio $(<1,1$ to $<1.25,1.25$ to $<2,2$ to $<4, \geq 4$ ), food security (yes/no), health insurance (government, private, multiple, none or single service), social support (yes/no), moderate or vigorous recreational physical activity (yes/ no), smoking status (current, former, never), and alcohol consumption (never, $<1 \mathrm{drink} / \mathrm{day},>1 \mathrm{drink} /$ day). Chosen race/ethnicity categories, including separation of Mexican Americans by birthplace, reflects previous findings that PFC concentrations differ across these categories [16]. In sensitivity analyses we further adjusted for aspects of metabolic syndrome, including hypercholesterolemia (yes/no), hypertension (yes/no), diabetes (yes/no) and body mass index $(<25,25$ to $<30, \geq 30)$, although these variables may be intermediates. The presence of hypercholesterolemia was defined as positive self-report, elevated measured serum cholesterol or reported use of medications commonly used to lower cholesterol. The presence of hypertension was likewise defined as positive self-report, elevated measured 
Table 1. Geometric mean (and geometric standard deviation) of PFC concentrations according to sample characteristics

\begin{tabular}{|c|c|c|c|c|c|c|}
\hline Characteristic & & $\mathrm{n}(\%)$ & \multicolumn{4}{|c|}{ Geometric mean (geometric standard deviation), $\mu \mathrm{g} / \mathrm{l}$} \\
\hline \multicolumn{2}{|l|}{ Total sample } & $1,766(100)$ & $22.63(2.13)$ & $4.08(1.97)$ & $1.01(2.35)$ & $2.05(2.30)$ \\
\hline \multirow[t]{3}{*}{ Age } & 60 to $<65$ years & $489(27.7)$ & $21.7(2.2)$ & $4.2(2.0)$ & $1.1(2.3)$ & $2.0(2.4)$ \\
\hline & 65 to $<69$ years & $399(22.6)$ & $23.0(2.1)$ & $4.2(2.0)$ & $1.1(2.3)$ & $2.1(2.2)$ \\
\hline & 70 to $<75$ years & $385(21.8)$ & $22.6(2.1)$ & $3.9(1.9)$ & $1.0(2.3)$ & $2.1(2.4)$ \\
\hline \multirow[t]{2}{*}{ Gender } & Male & $912(51.6)$ & $24.5(2.0)$ & $4.0(2.0)$ & $1.0(2.4)$ & $2.1(2.3)$ \\
\hline & Female & $854(48.4)$ & $20.8(2.2)$ & $4.1(2.0)$ & $1.0(2.3)$ & $2.0(2.4)$ \\
\hline \multirow[t]{4}{*}{ Race/ethnicity } & White & $931(52.7)$ & $23.1(2.1)$ & $4.4(1.9)$ & $1.0(2.2)$ & $2.1(2.3)$ \\
\hline & Black & $325(18.4)$ & $30.4(2.2)$ & $4.3(2.1)$ & $1.6(2.1)$ & $2.3(2.3)$ \\
\hline & Non-Mexican American Hispanic & $90(5.1)$ & $17.5(1.9)$ & $4.4(1.7)$ & $1.2(2.0)$ & $2.5(2.3)$ \\
\hline & Mexican American, not born in Mexico & $182(10.3)$ & $20.6(1.9)$ & $3.5(1.8)$ & $0.6(2.3)$ & $1.8(2.0)$ \\
\hline \multirow{2}{*}{ NHANES cycle } & $2005-2006$ & $417(23.6)$ & $23.0(2.3)$ & $4.2(2.2)$ & $1.2(2.2)$ & $1.8(2.5)$ \\
\hline & 2007-2008 & $493(27.9)$ & $17.9(2.1)$ & $4.6(1.7)$ & $1.6(1.9)$ & $2.5(2.2)$ \\
\hline \multirow[t]{4}{*}{ Education } & Less than high school & $747(42.3)$ & $22.5(2.2)$ & $3.8(2.0)$ & $0.9(2.5)$ & $1.9(2.3)$ \\
\hline & High school graduate & $417(23.6)$ & $22.9(2.0)$ & $4.3(2.0)$ & $1.1(2.3)$ & $2.1(2.3)$ \\
\hline & Some college & $345(19.5)$ & $22.6(2.1)$ & $4.4(1.9)$ & $1.0(2.1)$ & $2.1(2.2)$ \\
\hline & College graduate & $257(14.6)$ & $22.6(2.2)$ & $4.3(1.8)$ & $1.2(2.2)$ & $2.4(2.3)$ \\
\hline \multirow{6}{*}{$\begin{array}{l}\text { Poverty-income } \\
\text { ratio }\end{array}$} & $<1$ & $267(15.1)$ & $20.7(2.2)$ & $3.4(2.0)$ & $0.9(2.5)$ & $1.8(2.3)$ \\
\hline & 1 to $<1.25$ & $192(10.9)$ & $22.2(2.2)$ & $3.7(2.2)$ & $0.9(2.4)$ & $1.8(2.1)$ \\
\hline & 1.25 to $<2$ & $367(20.8)$ & $22.2(2.1)$ & $4.1(2.0)$ & $1.0(2.4)$ & $2.0(2.4)$ \\
\hline & 2 to $<4$ & $447(25.3)$ & $23.8(2.1)$ & $4.3(2.0)$ & $1.1(2.2)$ & $2.2(2.3)$ \\
\hline & $\geq 4$ & $345(19.5)$ & $23.8(2.1)$ & $4.5(1.8)$ & $1.2(2.2)$ & $2.3(2.2)$ \\
\hline & Missing & $148(8.4)$ & $21.9(2.1)$ & $4.2(1.9)$ & $1.0(2.3)$ & $1.9(2.5)$ \\
\hline $\begin{array}{l}\text { Household food } \\
\text { security }\end{array}$ & Missing & $36(2.0)$ & $28.9(2.3)$ & $3.5(2.4)$ & $0.9(2.5)$ & $2.2(2.4)$ \\
\hline \multirow[t]{3}{*}{ Social support } & Yes & $1,604(90.8)$ & $22.8(2.1)$ & $4.1(2.0)$ & $1.0(2.3)$ & $2.1(2.3)$ \\
\hline & No & $158(9.0)$ & $20.4(2.0)$ & $3.4(2.1)$ & $0.9(2.4)$ & $1.8(2.3)$ \\
\hline & Missing & $4(0.2)$ & $39.7(2.8)$ & $6.2(1.8)$ & $1.4(3.9)$ & $2.7(1.8)$ \\
\hline \multirow{2}{*}{$\begin{array}{l}\text { Recreational } \\
\text { physical activity }\end{array}$} & Yes & $751(42.5)$ & $23.0(2.1)$ & $4.2(1.9)$ & $1.1(2.2)$ & $2.2(2.3)$ \\
\hline & No & $1,015(57.5)$ & $22.4(2.1)$ & $4.0(2.0)$ & $1.0(2.4)$ & $1.9(2.3)$ \\
\hline \multirow[t]{4}{*}{ Smoking } & Never smoker & $811(45.9)$ & $22.6(2.2)$ & $4.1(1.9)$ & $1.0(2.4)$ & $2.1(2.3)$ \\
\hline & Past smoker & $716(40.5)$ & $22.1(2.1)$ & $3.9(2.0)$ & $1.0(2.3)$ & $2.0(2.3)$ \\
\hline & Current smoker & $238(13.5)$ & $24.4(2.1)$ & $4.3(2.0)$ & $1.1(2.3)$ & $2.2(2.1)$ \\
\hline & Missing & $1(0.1)$ & $22.4(-)$ & $3.4(-)$ & $0.3(-)$ & $1.7(-)$ \\
\hline \multirow[t]{4}{*}{ Alcohol use } & Never & $976(55.3)$ & $22.3(2.2)$ & $4.0(2.0)$ & $1.0(2.4)$ & $2.0(2.3)$ \\
\hline & $\leq 1$ drink per day & $591(33.5)$ & $23.2(2.0)$ & $4.3(1.9)$ & $1.1(2.2)$ & $2.2(2.4)$ \\
\hline & $>1$ drink per day & $113(6.4)$ & $26.2(2.1)$ & $4.5(1.8)$ & $1.1(2.2)$ & $2.3(1.9)$ \\
\hline & Missing & $86(4.9)$ & $18.4(2.6)$ & $3.2(2.2)$ & $0.9(2.6)$ & $1.7(2.1)$ \\
\hline \multirow[t]{5}{*}{ Diabetes } & Yes, no diabetes medication use & $54(3.1)$ & $19.5(2.1)$ & $3.3(2.5)$ & $0.9(2.4)$ & $1.7(3.0)$ \\
\hline & Yes, nonthiazolidinedione medication use & $255(14.4)$ & $19.9(2.5)$ & $3.4(2.2)$ & $1.0(2.5)$ & $1.7(2.5)$ \\
\hline & Yes, thiazolidinedione medication use & $68(3.9)$ & $22.6(2.3)$ & $3.6(1.9)$ & $1.0(2.3)$ & $1.8(2.1)$ \\
\hline & No & $1,386(78.5)$ & $23.4(2.0)$ & $4.3(1.9)$ & $1.0(2.3)$ & $2.2(2.2)$ \\
\hline & Missing & $3(0.2)$ & $10.5(2.0)$ & $0.9(7.1)$ & $0.6(2.2)$ & $1.1(1.5)$ \\
\hline
\end{tabular}


Table 2. Association between a doubling in PFC concentrations and self-reported limitation due to difficulty remembering or periods of confusion $(n=1,766)$

\begin{tabular}{ll}
\hline & OR $(95 \% \mathrm{CI})^{\mathrm{a}}$ \\
\hline PFOS & $0.90(0.78,1.03)$ \\
PFOA & $0.92(0.78,1.09)$ \\
PFNA & $0.91(0.79,1.04)$ \\
PFHxS & $0.93(0.82,1.06)$ \\
\hline
\end{tabular}

a Adjusted for age, race/ethnicity, gender, education, NHANES cycle, poverty-income ratio, food security, health insurance status, social support, physical activity, alcohol consumption and smoking status.

blood pressure or use of antihypertensive medications. We used self-report and medication use data to define the presence or absence of diabetes in a similar fashion. We also explored adjusting for osmolality (continuous), estimated glomerular filtration rate [17] (continuous) and urinary albumin $(>/ \leq 80 \mu \mathrm{g} / \mathrm{dl})$ to investigate the potential for artifact, which would be introduced if poor cognition was associated with changes in serum volume or kidney function (e.g. as complications of diabetes) that might alter measured PFC serum concentrations. Finally, we evaluated the potential for confounding by fish consumption (yes/no) in the past 30 days in sensitivity analyses as data on fish consumption was unavailable for participants from the 2003-2004 cycle (missing fish consumption data were imputed, along with missing data on all other covariates).

For our primary analyses, we used SAS PROC SURVEYLOGISTIC, in line with NHANES analytical guidelines, to assess the associations between each PFC and self-reported limitation due to difficulty remembering or periods of confusion in separate models. However, we adjusted for covariates known to be included in the estimation of the NHANES sampling weights instead of weighting by the sampling weights, as this method provides a good balance between efficiency and bias in the analysis of complex survey data [18] and has been previously used in the analysis of NHANES data [19]. We addressed differences between the weighted and unweighted analyses through sensitivity analyses. We conducted standard diagnostics to evaluate model fit and evaluated the shape of the dose-response curve using transformations and restricted cubic splines. We also evaluated potential effect modification by age, gender and diabetes status/medication use using multiplicative interaction terms.

We used similar methods for analyses of the association between PFCs and our secondary outcome measures with the following differences: (a) we assessed the associations between PFCs and continuous DSST scores using PROC SURVEYREG and (b) we were only able to adjust for a subset of covariates (age, gender, abbreviated race/ethnicity, [i.e. non-Hispanic White, non-Hispanic Black, Hispanic, other ], cycle, and education) in analyses of the association between PFCs and self-reported difficulty with activities of daily living due to senility given the small number of cases. All analyses were completed in SAS, version 9.2 or R, version 2.13.0.

\section{Results}

We evaluated the association between PFCs and selfreported limitation due to difficulty remembering or periods of confusion in 1,766 individuals (mean age, 70.3; standard deviation, 6.8). 230 persons (13\%) reported experiencing limitation due to difficulty remembering or periods of confusion. Study sample characteristics prior to the imputation of missing data and the distribution of PFCs across characteristics, including diabetic status and diabetic medication use, are presented in table 1. After evaluating the shape of the dose-response curve the PFC concentrations were log transformed for use in all analyses and we report associations for a doubling in PFC concentration.

Our primary analyses suggest that there may be a protective association between PFC exposure and self-reported limitation due to difficulty remembering or periods of confusion (table 2). Sensitivity analyses show that these effect estimates are similar after additional adjustment for aspects of metabolic syndrome or fish consumption, and there was no evidence to support the idea that our results are due to artifact introduced by changes in serum volume or kidney function associated with cognition (online suppl. table; for all online suppl. material, see www. karger.com/doi/10.1159/000342310). Weighted analyses were similar in direction and magnitude, with the exception of the association with PFOA (which was null), but were less precise, as expected (online suppl. table). We found a similar pattern of association between PFCs and self-reported difficulties with activities of daily living due to senility in secondary analyses, but there was little evidence to support an association between PFCs and DSST scores (table 3 ).

We found no evidence of effect modification by age or gender. However, the association between PFCs and self-reported limitation due to difficulty remembering or periods of confusion appears to be modified by diabetic status and medication use. Specifically, the protective association appeared to be concentrated in diabetics for PFOS, PFOA and PFNA (table 4). Further stratification by medication use shows the association is strongest in nonmedicated diabetics (table 5), although the number of persons in this group is small $(\mathrm{n}=54)$, and there was little evidence for an association in diabetics taking thiazolidinedione medications. Adjustment for estimated glomerular filtration rate, osmolality and urinary albumin did not appreciably change these estimates (data not shown). 
Table 3. Association between a doubling in PFC concentrations and self-reported difficulties with activities of daily living due to senility $(n=1,766)$ or DSST scores $(n=275)$

\begin{tabular}{lll}
\hline & $\begin{array}{l}\text { Senility } \\
\text { OR }(95 \% \mathrm{CI})^{\mathrm{a}}\end{array}$ & $\begin{array}{l}\text { DSST } \\
\text { beta }(95 \% \mathrm{CI})^{\mathrm{b}}\end{array}$ \\
\hline PFOS & $0.83(0.60,1.16)$ & $-0.20(-1.89,1.50)$ \\
PFOA & $0.81(0.55,1.18)$ & $1.00(-0.87,2.87)$ \\
PFNA & $0.92(0.59,1.44)$ & $0.29(-1.69,2.26)$ \\
PFHxS & $0.77(0.57,1.05)$ & $0.39(-0.50,1.29)$ \\
\hline
\end{tabular}

a Adjusted for age, abbreviated race/ethnicity (non-Hispanic White, non-Hispanic Black, Hispanic, other), gender, NHANES cycle and education.

${ }^{b}$ Adjusted for age, race/ethnicity, gender, education, NHANES cycle, poverty-income ratio, food security, health insurance status, social support, physical activity, alcohol consumption and smoking status.
Table 4. Association between a doubling in PFC concentrations and self-reported limitation due to difficulty remembering or periods of confusion by diabetic status $(n=1,766)$

\begin{tabular}{llll}
\hline & OR $(95 \% \mathrm{CI})^{\mathrm{a}}$ & $\begin{array}{l}\text { p value for } \\
\text { interaction }\end{array}$ \\
\cline { 2 - 3 } & nondiabetics & diabetics & \\
\hline PFOS & $0.98(0.85,1.14)$ & $0.80(0.64,1.00)$ & 0.10 \\
PFOA & $1.04(0.89,1.22)$ & $0.80(0.60,1.08)$ & 0.10 \\
PFNA & $0.98(0.84,1.14)$ & $0.77(0.59,1.00)$ & 0.14 \\
PFHxS & $0.95(0.84,1.09)$ & $0.95(0.73,1.22)$ & 0.97 \\
\hline
\end{tabular}

${ }^{a}$ Adjusted for age, race/ethnicity, gender, NHANES cycle, education, poverty-income ratio, food security, health insurance status, social support, physical activity, alcohol consumption and smoking status.

Table 5. Association between a doubling in PFC concentrations and self-reported limitation due to difficulty remembering or periods of confusion by diabetic status and diabetic medication use $(n=1,766)$

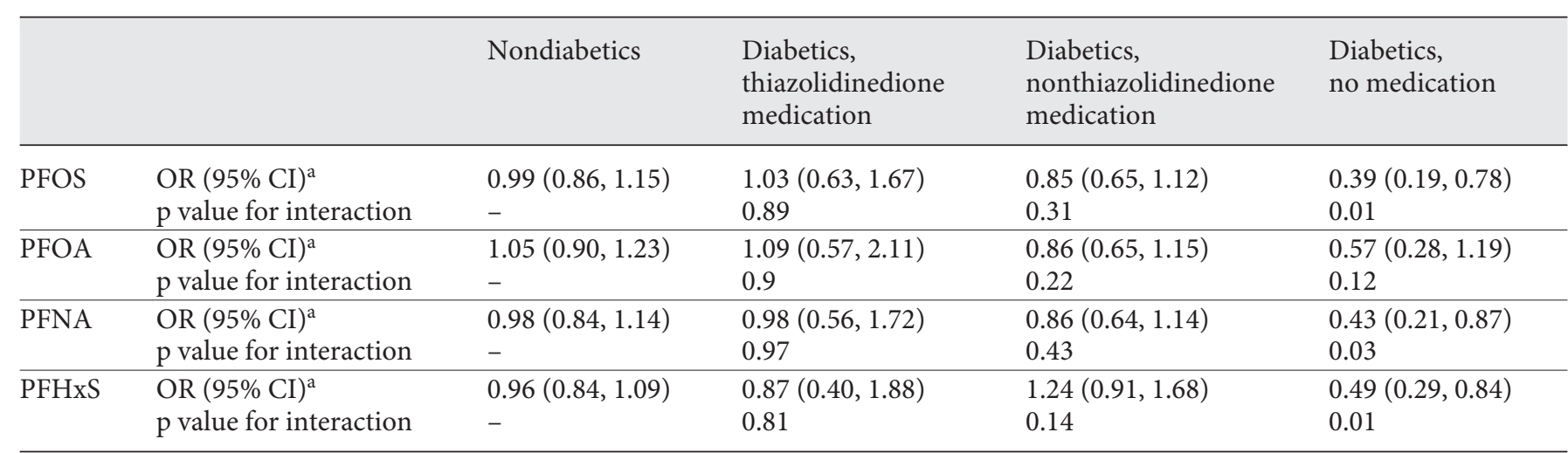

a Adjusted for age, race/ethnicity, gender, NHANES cycle, education, poverty-income ratio, food security, health insurance status, social support, physical activity, alcohol consumption and smoking status.

\section{Discussion}

This cross-sectional study supports the hypothesis that PFCs may be neuroprotective. Our data suggest that higher PFC concentrations are associated with reduced risk of cognitive limitation, especially among nonmedicated diabetics.

Our current mechanistic hypothesis posits that PFCs may exert neuroprotective effects via the activation of PPAR- $\gamma$, as PFOA and PFOS have been shown to activate human PPAR- $\alpha$ and PPAR- $\gamma$ under some experimental conditions $[3,20,21]$. Other PPAR- $\gamma$ agonists, which in- clude nonsteroidal anti-inflammatory drugs and the antidiabetic thiazolidinedione drugs, appear to be neuroprotective [22-28], potentially through the inhibition of inflammation, oxidative stress and apoptosis, modulation of signaling pathways involved in the pathogenesis of neurodegenerative diseases, or improvement in insulin sensitivity and reduction in blood glucose levels [4]; however, it must be recognized that they may also act exclusive of PPAR- $\gamma$ activation. In our study, we observed the strongest protective associations of PFCs on limitation due to difficulty remembering or periods of confusion within nonmedicated diabetics. Conversely, we found no 
association in nondiabetics or diabetics using thiazolidinedione drugs for all PFCs and a moderate protective association in persons using only nonthiazolidinedione diabetes medications for three of the four PFCs. This pattern supports the idea that PFCs and thiazolidinedione drugs may share a common mechanism, PPAR- $\gamma$ activation.

The finding that the association was concentrated in diabetics, specifically nonmedicated diabetics, raises the question of whether diabetes or diabetic severity influences PFC concentrations. Hypothetically, diabetic nephropathy may impact PFC concentrations. First, if PFCs are excreted via urine, a lower glomerular filtration rate could artificially raise the serum PFC concentrations. However, this would induce an adverse association given that the increasing severity of diabetes would predict both higher PFC levels and worse cognition. Second, kidney dysfunction could lead to either increased water retention and higher plasma volume or dehydration due to water loss associated with loss of glucose in the urine and lower plasma volume. Higher plasma volume could artificially lower PFC concentrations in persons with more severe diabetes, inducing a protective association, while lower plasma volume could be expected to do the opposite. Third, if excess PFCs are lost because of their association with serum protein, as poor kidney function leads to protein loss in urine, PFC concentrations could be lower in diabetics with greater diabetic severity, artificially inducing a protective association. However, adjustment for osmolality (a marker of plasma concentration), estimated glomerular filtration rate (a marker of kidney function) and urinary albumin (reflecting protein loss) did not appreciably change our estimates, suggesting that such explanations are unlikely to account for the observed associations.

We recognize that our primary measure of cognition, self-reported limitation due to difficulty remembering or periods of confusion, is not a clinical endpoint. However, in clinical settings, subjective memory complaint often motivates clinical evaluation of cognition and is generally accepted as a key feature of mild cognitive impairment [29]. Indeed, a growing body of literature has linked subjective memory complaint prospectively to poor cognition [30], dementia [31], neuropathology [32] and future cognitive decline $[30,33]$. Our measure, self-reported limitation due to poor memory or periods of confusion, is a strengthened version of questions about subjective memory impairment/complaints given that it focuses on limitations due to poor memory or confusion, rather than difficulty remembering or poor memory compared with previous performance [34], and so is more likely to reflect current impairment. Supporting this, the prevalence of subjective memory complaints is typically about $20-60 \%$ [33], while the prevalence of cognitive limitation in our data is $13 \%$. In addition, variables shown in other studies to predict cognitive impairment or cognitive decline, such as age, diabetes, physical activity and hypertension, also appear to predict our outcome in multivariable models (data not shown). Further research should evaluate the association between PFCs and cognitive test scores or clinical diagnoses.

The relevant period of potential exposure susceptibility is difficult to identify, but our understanding of cognitive decline suggests a period of years to decades prior to the clinical onset of dementia. More recent exposures may influence the progression of brain pathology underlying cognitive difficulties, while more distant exposures may influence initiation of this pathology. The assumption that current PFC concentrations reflect past concentrations in a relevant time period for exposure is reasonable given their long half-life in humans, ranging from 2.3 years for PFOA to 8 years for PFHxS $[35,36]$.

The remaining limitations of our study must be noted. We did not find support for an association between PFCs and DSST scores; however, these analyses were limited by the small number of participants and the limited range of cognitive domains assessed by the DSST. As our study is cross-sectional, it is possible that poor or declining cognition impacts PFC levels, rather than the reverse, but this explanation seems unlikely given the long half-life of PFCs in humans. The potential for bias due to residual or unmeasured confounding remains. In particular, we were only able to adjust for fish consumption in sensitivity analyses; however, results from our sensitivity analyses were consistent with our main findings. We were able to control for many known or suspected confounders, including sociodemographic factors. Selection bias due to nonparticipation is not expected to influence our results given that people are unaware of their PFC exposures and PFCs are not known to significantly predict mortality. Misclassification of PFC exposure or cognitive impairment may contribute to the current findings, making it more difficult to detect a true effect. Finally, we were unable to disentangle the effects of individual PFCs, as all four are moderately correlated [range, 0.35 (PFNA and PFHxS) to 0.64 (PFOA and PFOS)]. However, to our knowledge, this is the first paper to consider the relationship between PFCs and cognition in older adults and we believe that the difficulties discussed above are unlikely to account for a protective association. 


\section{Conclusion}

This cross-sectional study supports the hypothesis that PFCs may have neuroprotective qualities, especially in diabetics. Confirmation of these findings in other data sets is needed and further exploration of the interplay between PFCs and human PPAR- $\gamma$ is warranted.

\section{Acknowledgements}

Melinda C. Power is supported by a National Institute of Aging training grant (1F31AG038233-01). Andrea A. Baccarelli is supported by New Investigator funding from the HSPH-NIEHS Center for Environmental Health (ES000002). Thomas Webster is supported in part by NIH grant R01ES015829.

\section{Disclosure Statement}

The authors have no conflicts of interest.

\section{References}

-1 Lau C, Anitole K, Hodes C, Lai D, PfahlesHutchens A, Seed J: Perfluoroalkyl acids: a review of monitoring and toxicological findings. Toxicol Sci 2007;99:366-394.

- 2 Fromme H, Tittlemier SA, Volkel W, Wilhelm M, Twardella D: Perfluorinated compounds - exposure assessment for the general population in western countries. Int J Hyg Environ Health 2009;212:239-270.

- 3 Vanden Heuvel JP, Thompson JT, Frame SR, Gillies PJ: Differential activation of nuclear receptors by perfluorinated fatty acid analogs and natural fatty acids: a comparison of human, mouse, and rat peroxisome proliferator-activated receptor-alpha, -beta, and -gamma, liver X receptor-beta, and retinoid X receptor-alpha. Toxicol Sci 2006;92:476489.

4 Kaundal RK, Sharma SS: Peroxisome proliferator-activated receptor gamma agonists as neuroprotective agents. Drug News Perspect 2010;23:241-256.

5 Steenland K, Fletcher T, Savitz DA: Epidemiologic evidence on the health effects of perfluorooctanoic acid (PFOA). Environ Health Perspect 2010;118:1100-1108.

-6 Corsini E, Avogadro A, Galbiati V, dell'Agli M, Marinovich M, Galli CL, Germolec DR: In vitro evaluation of the immunotoxic potential of perfluorinated compounds (PFCs). Toxicol Appl Pharmacol 2011;250:108-116.

-7 Grandjean P, Andersen EW, Budtz-Jørgensen E, Nielsen F, Mølbak K, Weihe P, Heilmann C: Serum vaccine antibody concentrations in children exposed to perfluorinated compounds. JAMA 2012;307:391-397.

$\checkmark 8$ Harada KH, Ishii TM, Takatsuka K, Koizumi A, Ohmori H: Effects of perfluorooctane sulfonate on action potentials and currents in cultured rat cerebellar purkinje cells. Biochem Biophys Res Commun 2006;351:240245.

9 Liu X, Liu W, Jin Y, Yu W, Liu L, Yu H: Effects of subchronic perfluorooctane sulfonate exposure of rats on calcium-dependent signaling molecules in the brain tissue. Arch Toxicol 2010;84:471-479.
10 Austin ME, Kasturi BS, Barber M, Kannan K, MohanKumar PS, MohanKumar SM: Neuroendocrine effects of perfluorooctane sulfonate in rats. Environ Health Perspect 2003;111:1485-1489.

11 Calafat AM, Wong LY, Kuklenyik Z, Reidy JA, Needham LL: Polyfluoroalkyl chemicals in the US Population: data from the National Health and Nutrition Examination Survey (NHANES) 2003-2004 and comparisons with NHANES 1999-2000. Environ Health Perspect 2007;115:1596-1602.

12 Kato K, Wong LY, Jia LT, Kuklenyik Z, Calafat AM: Trends in exposure to polyfluoroalkyl chemicals in the US population: 19992008. Environ Sci Technol 2011;45:80378045.

13 van Wijngaarden E, Winters PC, CorySlechta DA: Blood lead levels in relation to cognitive function in older US adults. Neurotoxicology 2011;32:110-115.

14 Wechsler D: Administration and Scoring Guide, Wechsler Adult Intelligence Scale Third Edition (WAIS-III). San Antonio, Harcourt Brace \& Co, 1997.

15 Allison PD: Imputation of categorical variables with PROC MI; in SAS Institute Inc (ed): Proceedings of the Thirtieth Annual SAS $^{\circledR}$ Users Group International Conference. Cary, SAS Institute Inc, 2005.

16 Nelson JW, Scammell MK, Hatch EE, Webster TF: Social disparities in exposures to bisphenol A and polyfluoroalkyl chemicals: a cross-sectional study within NHANES 2003-2006. Environ Health 2012;11:10.

17 Levey AS, Stevens LA, Schmid CH, Zhang YL, Castro AF, 3rd, Feldman HI, Kusek JW, Eggers P, Van Lente F, Greene T, Coresh J: A new equation to estimate glomerular filtration rate. Ann Intern Med 2009;150:604612.

18 Korn EL, Graubard BI: Epidemiologic studies utilizing surveys: accounting for the sampling design. Am J Public Health 1991;81: 1166-1173.

19 Nelson JW, Hatch EE, Webster TF: Exposure to polyfluoroalkyl chemicals and cholester- ol, body weight, and insulin resistance in the general US population. Environ Health Perspect 2010;118:197-202.

20 Takacs ML, Abbott BD: Activation of mouse and human peroxisome proliferator-activated receptors (alpha, beta/delta, gamma) by perfluorooctanoic acid and perfluorooctane sulfonate. Toxicol Sci 2007;95:108-117.

21 Maloney EK, Waxman DJ: Trans-activation of PPAR-alpha and PPAR-gamma by structurally diverse environmental chemicals. Toxicol Appl Pharmacol 1999;161:209-218.

-22 Szekely CA, Green RC, Breitner JC, Ostbye T, Beiser AS, Corrada MM, Dodge HH, Ganguli M, Kawas CH, Kuller LH, Psaty BM, Resnick SM, Wolf PA, Zonderman AB, Welsh-Bohmer KA, Zandi PP: No advantage of A beta 42-lowering NSAIDs for prevention of Alzheimer dementia in six pooled cohort studies. Neurology 2008;70:2291-2298.

23 Geldmacher DS, Fritsch T, McClendon MJ Landreth G: A randomized pilot clinical trial of the safety of pioglitazone in treatment of patients with Alzheimer disease. Arch Neurol 2011;68:45-50.

24 Gold M, Alderton C, Zvartau-Hind M, Egginton S, Saunders AM, Irizarry M, Craft S, Landreth G, Linnamagi U, Sawchak S: Rosiglitazone monotherapy in mild-to-moderate Alzheimer's disease: results from a randomized, double-blind, placebo-controlled phase III study. Dement Geriatr Cogn Disord 2010; 30:131-146.

25 Risner ME, Saunders AM, Altman JF, Ormandy GC, Craft S, Foley IM, Zvartau-Hind ME, Hosford DA, Roses AD: Efficacy of rosiglitazone in a genetically defined population with mild-to-moderate Alzheimer's disease. Pharmacogenomics J 2006;6:246-254.

26 Watson GS, Cholerton BA, Reger MA, Baker LD, Plymate SR, Asthana S, Fishel MA, Kulstad JJ, Green PS, Cook DG, Kahn SE, Keeling ML, Craft S: Preserved cognition in patients with early Alzheimer disease and amnestic mild cognitive impairment during treatment with rosiglitazone: a preliminary study. Am J Geriatr Psychiatry 2005;13:950-958. 
27 Hanyu H, Sato T, Kiuchi A, Sakurai H, Iwamoto T: Pioglitazone improved cognition in a pilot study on patients with Alzheimer's disease and mild cognitive impairment with diabetes mellitus. J Am Geriatr Soc 2009;57: 177-179.

-28 Sato T, Hanyu H, Hirao K, Kanetaka H, Sakurai $\mathrm{H}$, Iwamoto T: Efficacy of PPAR- $\gamma$ agonist pioglitazone in mild alzheimer disease. Neurobiol Aging 2011;32:1626-1633.

-29 Winblad B, Palmer K, Kivipelto M, Jelic V, Fratiglioni L, Wahlund LO, Nordberg A, Backman L, Albert M, Almkvist O, Arai H, Basun H, Blennow K, de Leon M, DeCarli C, Erkinjuntti T, Giacobini E, Graff C, Hardy J, Jack C, Jorm A, Ritchie K, van Duijn C, Visser P, Petersen RC: Mild cognitive impairment - beyond controversies, towards a consensus: report of the International Working Group on Mild Cognitive Impairment. J Intern Med 2004;256:240-246.
30 Jorm AF, Christensen H, Korten AE, Jacomb PA, Henderson AS: Memory complaints as a precursor of memory impairment in older people: a longitudinal analysis over 7-8 years. Psychol Med 2001;31:441-449.

31 Mitchell AJ: The clinical significance of subjective memory complaints in the diagnosis of mild cognitive impairment and dementia: a meta-analysis. Int J Geriatr Psychiatry 2008;23:1191-1202.

32 Miranda B, Madureira S, Verdelho A, Ferro J, Pantoni L, Salvadori E, Chabriat H, Erkinjuntti T, Fazekas F, Hennerici M, O’Brien J, Scheltens P, Visser MC, Wahlund LO, Waldemar G, Wallin A, Inzitarion D: Selfperceived memory impairment and cognitive performance in an elderly independent population with age-related white matter changes. J Neurol Neurosurg Psychiatry 2008;79:869-873.
33 Jonker C, Geerlings MI, Schmand B: Are memory complaints predictive for dementia? A review of clinical and populationbased studies. Int J Geriatr Psychiatry 2000; 15:983-991.

34 Abdulrab K, Heun R: Subjective memory impairment. A review of its definitions indicates the need for a comprehensive set of standardised and validated criteria. Eur Psychiatry 2008;23:321-330.

35 Olsen GW, Burris JM, Ehresman DJ, Froehlich JW, Seacat AM, Butenhoff JL, Zobel LR: Half-life of serum elimination of perfluorooctanesulfonate, perfluorohexanesulfonate, and perfluorooctanoate in retired fluorochemical production workers. Environ Health Perspect 2007;115:1298-1305.

36 Bartell SM, Calafat AM, Lyu C, Kato K, Ryan $\mathrm{PB}$, Steenland K: Rate of decline in serum PFOA concentrations after granular activated carbon filtration at two public water systems in Ohio and West Virginia. Environ Health Perspect 2010;118:222-228. 